\title{
Optimal Entanglement Witnesses: A Scalable Data-Driven Approach
}

\author{
Irénée Frérot $\oplus^{1,2, *}$ and Tommaso Roscilde ${ }^{3, \dagger}$ \\ ${ }^{1}$ ICFO-Institut de Ciencies Fotoniques, The Barcelona Institute of Science and Technology, \\ Avenida Carl Friedrich Gauss 3, 08860 Barcelona, Spain \\ ${ }^{2}$ Max-Planck-Institut für Quantenoptik, D-85748 Garching, Germany \\ ${ }^{3}$ Laboratoire de Physique, CNRS UMR 5672, Ecole Normale Supérieure de Lyon, Université de Lyon, \\ 46 Allée d'Italie, Lyon F-69364, France
}

(Received 14 January 2021; revised 21 April 2021; accepted 17 June 2021; published 20 July 2021)

\begin{abstract}
Multipartite entanglement is a key resource allowing quantum devices to outperform their classical counterparts, and entanglement certification is fundamental to assess any quantum advantage. The only scalable certification scheme relies on entanglement witnessing, typically effective only for special entangled states. Here, we focus on finite sets of measurements on quantum states (hereafter called quantum data), and we propose an approach which, given a particular spatial partitioning of the system of interest, can effectively ascertain whether or not the dataset is compatible with a separable state. When compatibility is disproven, the approach produces the optimal entanglement witness for the quantum data at hand. Our approach is based on mapping separable states onto equilibrium classical field theories on a lattice and on mapping the compatibility problem onto an inverse statistical problem, whose solution is reached in polynomial time whenever the classical field theory does not describe a glassy system. Our results pave the way for systematic entanglement certification in quantum devices, optimized with respect to the accessible observables.
\end{abstract}

DOI: 10.1103/PhysRevLett.127.040401

Introduction.-Preparing and processing strongly entangled many-body states, in both a controlled and scalable way, is the goal of all quantum simulators and computers. Indeed, as the efficient representation of generic entangled many-body states is impossible on classical machines, entanglement represents the key computational resource of these devices [1,2]. As a consequence, developing generic and scalable methods to certify entanglement in multipartite systems stands as a grand challenge of quantum information science. Even more fundamentally, entanglement certification is a central task to probe the resilience of quantum correlations from the microscopic world to the macroscopic one [3].

Any practical method must circumvent the tomographic reconstruction of the full density matrix [4,5] (which implies a number of measurements scaling exponentially with system size), and it should instead infer entanglement from the partial information contained in a given dataset of measurement results (hereafter referred to as quantum data). When one adopts this data-driven strategy, the goal of entanglement certification is to establish whether or not

Published by the American Physical Society under the terms of the Creative Commons Attribution 4.0 International license. Further distribution of this work must maintain attribution to the author(s) and the published article's title, journal citation, and DOI. Open access publication funded by the Max Planck Society. the quantum data are compatible with a separable state [5-7]. Given an extended quantum system composed of $N_{\text {tot }}$ degrees of freedom, grouped together into $N \leq N_{\text {tot }}$ clusters [see Fig. 1(a)], the state $\hat{\rho}$ of the system is separable [8] if it can be written in the form

$$
\hat{\rho}_{p}:=\int d \lambda p(\lambda) \hat{\rho}_{\text {prod }}(\lambda)
$$

where $\hat{\rho}_{\text {prod }}(\lambda)=\bigotimes_{i=1}^{N}\left|\psi_{i}\left(\lambda_{i}\right)\right\rangle\left\langle\psi_{i}\left(\lambda_{i}\right)\right|$ is a product state of the partition, $\left|\psi_{i}\left(\lambda_{i}\right)\right\rangle$ being the state of the $i$ th cluster, parametrized by parameters $\lambda=\left(\lambda_{1}, \ldots, \lambda_{i}, \ldots, \lambda_{N}\right)$, distributed according to $p(\lambda) \geq 0$ [Fig. 1(b)]. The distribution $p$ fully specifies classical correlations across the partition. A multipartite entangled state $\hat{\rho}$, on the other hand, cannot be written in the above form. Given a set of observables $\hat{A}_{a}$ $(a=1, \ldots, R)$, multipartite entanglement is therefore witnessed by the quantum dataset $\left\{\left\langle\hat{A}_{a}\right\rangle_{\hat{\rho}}\right\}_{a=1}^{R}$ [where $\left\langle\hat{A}_{a}\right\rangle_{\hat{\rho}}=$ $\left.\operatorname{Tr}\left(\hat{A}_{a} \hat{\rho}\right)\right]$ if one proves that the latter cannot be reproduced by any separable state. This task is accomplished by proving that the quantum data violate an entanglement witness (EW) inequality, $\langle\hat{\mathcal{W}}\rangle_{\hat{\rho}_{p}}=\sum_{a} W_{a}\left\langle\hat{A}_{a}\right\rangle_{\hat{\rho}_{p}} \geq B_{\text {sep }}$, valid for all separable states $\hat{\rho}_{p}$ [9]. Here, $W_{a}$ are suitable coefficients, and $B_{\text {sep }}$ is the so-called separable bound.

EW operators $\hat{\mathcal{W}}$ are generally defined based on the properties of special entangled states (e.g., squeezed states, total spin singlets, etc.) [9], and failure of a dataset to 

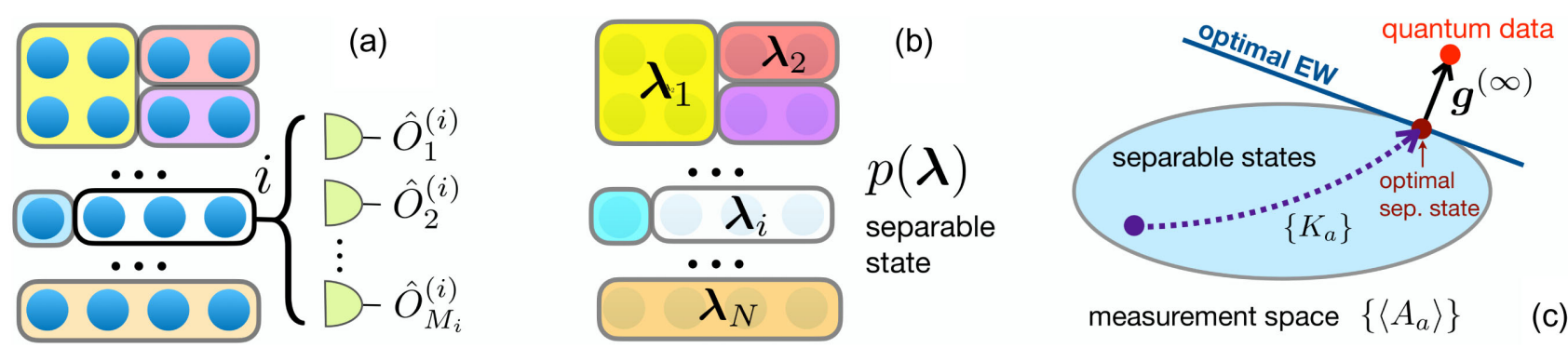

FIG. 1. (a) Partition of a quantum device into $N$ clusters, each of which is subject to $M_{i}$ measurements. (b) A separable state of the system is described as a probability distribution $p(\lambda)$ of local states defined by the $\left\{\lambda_{i}\right\}$ parameters. (c) Our algorithm builds a trajectory of separable states [parametrized by couplings $\left\{K_{a}\right\}$ defining $p(\lambda)$ ] which converges to the optimal state approximating at best some target quantum data. If the state fails to reproduce the quantum data exactly, the vector joining the optimal separable data and the quantum data reconstructs the optimal EW inequality.

violate a given EW inequality does not exclude the existence of a different violated inequality involving the same data, yet to be discovered. This may erroneously suggest that entanglement witnessing is limited by creativity and physical insight and that the entanglement witnessing problem ("is a quantum dataset compatible with a separable state?") [5-7] is generically undecidable. The goal of our work is to show that this is not the case and that the entanglement witnessing capability of a quantum dataset can be exhaustively tested. Our key insight is that the problem of finding the distribution $p(\lambda)$, which defines the separable state reproducing at best the quantum data, is a statistical inference problem; and, remarkably, it has the structure of a convex optimization problem, whose solution can be attained in a time scaling polynomially with the partition size (under mild assumptions) and with the Hilbert space dimension of the subsystems composing the partition. When the optimal separable state fails to reproduce the quantum data, the distance between the quantum dataset $\left\{\left\langle\hat{A}_{a}\right\rangle_{\hat{\rho}}\right\}$ and the optimal separable set $\left\{\left\langle\hat{A}_{a}\right\rangle_{\hat{\rho}_{p}}\right\}$ allows one to reconstruct the optimal $\mathrm{EW}$ inequality violated by the quantum data [Fig. 1(c)]. We benchmark our approach by establishing new EW inequalities satisfied by the lowtemperature states of the Heisenberg antiferromagnetic chain and the quantum Ising chain; in the latter case, our new EW inequalities outperform all previously known EW criteria for multipartite entanglement. Our work parallels the recent mapping of the Bell-nonlocality detection problem onto an inverse statistical problem [10], and it offers an efficient scheme for entanglement detection in state-of-the-art quantum devices within a device-dependent scenario.

Quantum dataset.-For definiteness, we assume that, on each subsystem $i=1, \ldots, N, M_{i}$ local observables $\hat{O}_{m}^{(i)}$ can be measured $\left(m=1, \ldots, M_{i}\right.$; e.g., the Pauli matrices $\hat{\sigma}_{a}^{(i)}$, $a \in\{x, y, z\}$ for individual qubits taken as subsystems) [Fig. 1(a)]. For convenience, we denote the local identity operator by $\hat{O}_{0}^{(i)}:=\mathbb{1}$. In order to reveal entanglement, these local observables must be noncommuting $\left(\left[\hat{O}_{m}^{(i)}, \hat{O}_{n}^{(i)}\right] \neq 0\right.$ for $1 \leq m<n \leq M_{i}$ ) [11]. From these local observables, we build $p$-body correlators of the form $\hat{O}_{m}=\bigotimes_{i=1}^{N} \hat{O}_{m_{i}}^{(i)}$, where $m_{i}=0$ for $N-p$ subsystems. Arbitrary observables can be built as linear combinations of correlators-such as, e.g., powers of collective spin variables $[12,13] \hat{J}_{a}=$ $\sum_{i} \hat{\sigma}_{a}^{(i)} / 2(a=x, y, z)$ for systems of qubits. Hence, we shall assume that $R$ observables of the form $\hat{A}_{a}=$ $\sum_{m} x_{m}^{(a)} \hat{O}_{m}$ can be measured, where the sum runs over all strings $\boldsymbol{m}=\left(m_{1}, \ldots, m_{N}\right)$ and $x_{\boldsymbol{m}}^{(a)}$ are arbitrary real coefficients. The quantum data $\left\{\left\langle\hat{A}_{a}\right\rangle_{\hat{\rho}}\right\}_{a=1}^{R}$ form the basis for entanglement certification in our scheme. The problem of entanglement certification based on a dataset has been discussed in the past, but the proposed methods either lack scalability [6] or are scalable only under some restrictive assumptions (short-range correlations, low-dimensional geometry) [7]. Our method aims at surpassing these limitations.

Mapping onto an inverse statistical problem.-The key aspect behind our approach is the limited information content of separable states. The parameters $\lambda$ specifying the product state $\hat{\rho}_{\text {prod }}(\lambda)$ can indeed be chosen as $\sum_{i}\left(2 d_{i}-2\right) \sim \mathcal{O}(N)$ real parameters, where $d_{i}$ is the dimension of the local Hilbert space of the $i$ th subsystem [14]. The average of the $\hat{A}_{a}$ observable on a separable state reads

$$
\left\langle\hat{A}_{a}\right\rangle_{\hat{\rho}_{p}}=\int d \lambda p(\lambda) \mathcal{A}_{a}(\lambda)=:\left\langle\mathcal{A}_{a}\right\rangle_{p},
$$

where $\quad \mathcal{A}_{a}(\lambda)=\sum_{m} x_{m}^{(a)} \prod_{i=1}^{N} o_{m_{i}}^{(i)}\left(\lambda_{i}\right) \quad$ and $\quad o_{m_{i}}^{(i)}\left(\lambda_{i}\right)=$ $\left\langle\psi_{i}\left(\lambda_{i}\right)\left|\hat{O}_{m_{i}}^{(i)}\right| \psi_{i}\left(\lambda_{i}\right)\right\rangle$. Given a product state, the calculation of each term in the sum defining $\mathcal{A}_{a}(\lambda)$ is clearly an operation scaling as $\mathcal{O}(N)$. Once the quantum nature of the state has been absorbed in $\mathcal{A}_{a}(\lambda)$, the calculation of $\left\langle\hat{A}_{a}\right\rangle_{\hat{\rho}_{p}}$ [Eq. (2)] is a classical statistical average over the distribution $p$ which, from a statistical physics viewpoint, can be regarded as the Boltzmann distribution $p(\lambda)=$ : $\exp [-H(\lambda)] / \mathcal{Z}$ of a classical field theory on a lattice 
(normalized by the $\mathcal{Z}$ factor), with a vector field $\lambda_{i}$ defined on each of the $N$ clusters [Fig. 1(b)]. The complexity of separable states is fundamentally inscribed in the effective Hamiltonian $H(\lambda)$, which is a priori arbitrary; namely, it is specified by a number $\mathcal{O}[\exp (N)]$ of parameters.

Once the classical statistical structure of the expectation values on separable states is exposed, the problem of reproducing the quantum data with a separable state takes the form of a statistical inference problem, whose solution is well known in statistical physics [15]. First of all, applying a maximum-entropy principle [16], the Hamiltonian can be parametrized without loss of generality with as many parameters as the elements of the quantum dataset [17]:

$$
H(\lambda)=-\sum_{a=1}^{R} K_{a} \mathcal{A}_{a}(\lambda)
$$

The parameters $\boldsymbol{K}=\left\{K_{a}\right\}_{a=1}^{R}$-the coupling constants of the classical field theory - are Lagrange multipliers whose optimization allows one to build the separable state $\hat{\rho}_{p}$ whose expectation values $\left\{\left\langle\hat{A}_{a}\right\rangle_{\hat{\rho}_{p}}\right\}$ best approximate the quantum data $\left\{\left\langle\hat{A}_{a}\right\rangle_{\hat{\rho}}\right\}$. The optimization of $\boldsymbol{K}$ can be efficiently achieved upon minimizing the cost function $L(\boldsymbol{K}):=\log \mathcal{Z}(\boldsymbol{K})-\sum_{a} K_{a}\left\langle\hat{A}_{a}\right\rangle_{\hat{\rho}}[10,15]$. The $a$-th component of the gradient of $L$ is $g_{a}:=\left(\partial L / \partial K_{a}\right)=\left\langle\mathcal{A}_{a}\right\rangle_{p}-$ $\left\langle\hat{A}_{a}\right\rangle_{\hat{\rho}}$, and its Hessian matrix is $\left(\partial^{2} L / \partial K_{a} \partial K_{b}\right)=$ $\left\langle\mathcal{A}_{a} \mathcal{A}_{b}\right\rangle_{p}-\left\langle\mathcal{A}_{a}\right\rangle_{p}\left\langle\mathcal{A}_{b}\right\rangle_{p}$, namely, the covariance matrix of the $\mathcal{A}_{a}(\lambda)$ functions. Since the latter is a semidefinite positive matrix, $L$ is a convex function. Therefore, a simple gradient-descent algorithm, which consists in iterating the update rule $K_{a}^{\prime}=K_{a}-\epsilon\left[\left\langle\mathcal{A}_{a}\right\rangle_{p}-\left\langle\hat{A}_{a}\right\rangle_{\hat{\rho}}\right]$ with $\epsilon \ll 1$, or any improvement thereof, is guaranteed to reach the global optimum of the problem. In practice, this requires one to repeatedly compute $\left\langle\mathcal{A}_{a}\right\rangle_{p}$ as in Eq. (2), a task efficiently accomplished, e.g., by Markov-chain Monte Carlo sampling of $p(\lambda)$, whenever the Hamiltonian $H$ does not describe a glassy system. The restriction to nonglassy systems is the only practical limitation of our approach [17] and is ensured in the examples considered below by considering translationally invariant systems.

Construction of an optimal entanglement witness.-As illustrated in Fig. 1(c), the algorithm converges to the distribution $p$ which minimizes $|\boldsymbol{g}|$-the norm of the gradient of $L$. If the minimal distance $g^{(\mathrm{min})}$ vanishes (within the error on the quantum data), i.e., if $\left\langle\hat{A}_{a}\right\rangle_{\hat{\rho}_{p}^{(\min )}}=$ $\left\langle\hat{A}_{a}\right\rangle_{\hat{\rho}}$ for all $a=1, \ldots, R$, then entanglement cannot be assessed from the available data. But in the opposite case, the coupling constants $K_{a}$ increase indefinitely along the optimization, and the coefficients of the gradient $g_{a}^{(\min )}=$ $\left\langle\hat{A}_{a}\right\rangle_{\hat{\rho}_{p}^{\text {(min) }}}-\left\langle\hat{A}_{a}\right\rangle_{\hat{\rho}}$ allow us to build a violated EW inequality. First, we define the normalized coefficients $W_{a}:=-g_{a}^{(\min )} /\left|\boldsymbol{g}^{(\mathrm{min})}\right|$. The condition $\left|\boldsymbol{g}^{(\mathrm{min})}\right|^{2}>0$ is then rewritten as

$$
-\sum_{a=1}^{R} W_{a}\left\langle\hat{A}_{a}\right\rangle_{\hat{\rho}}<\min _{\hat{\rho}_{p}}\left\{-\sum_{a=1}^{R} W_{a}\left\langle\hat{A}_{a}\right\rangle_{\hat{\rho}_{p}}\right\}=: B_{\text {sep }} .
$$

The linear combination $\hat{\mathcal{W}}:=-\sum_{a=1}^{R} W_{a} \hat{A}_{a}$ is the datadriven EW operator. The separable bound $B_{\text {sep }}$, namely, the minimal value of $\operatorname{Tr}(\hat{\rho} \hat{\mathcal{W}})$ over separable states, is violated by the dataset, ultimately proving that entanglement is present among the subsystems. The operator $\hat{\mathcal{W}}$ is optimal, in that any other normalized linear combination $\hat{\mathcal{W}}^{\prime}=$ $-\sum_{a} W_{a}^{\prime} \hat{A}_{a}$ [with $\sum_{a}\left(W_{a}^{\prime}\right)^{2}=1$ ] defines an EW inequality whose violation cannot exceed the violation of the inequality involving $\hat{\mathcal{W}}$. This property follows from the convexity of the set of separable states.

Complexity of the algorithm.-If the quantum data contain correlation functions involving up to $k$ points, the effective Hamiltonian $H$ contains $\mathcal{O}\left(N^{k}\right)$ terms; therefore, the computational cost of evaluating statistical averages of the kind of Eq. (2) with a precision of $\epsilon$ (using Monte Carlo sampling) scales as $\mathcal{O}\left(d^{2} \epsilon^{-2} N^{k}\right)$, where $\mathcal{O}\left(d^{2}\right)$ is the cost of evaluating the local observables $o_{m_{i}}^{(i)}\left(\lambda_{i}\right)$ when $d_{i}=d$. The polynomial scaling of the computational cost with the number $N$ of parties and with the local Hilbert space dimension is the central asset of our approach.

Ensembles of qubits.-Hereafter, we shall specify our attention to the case of systems of $N$ qubits partitioned into subsystems consisting of single qubits; and quantum data will be assumed to consist of one- and two-point correlations, $\left\langle\hat{\sigma}_{a}^{(i)}\right\rangle_{\hat{\rho}}$ and $\left\langle\hat{\sigma}_{a}^{(i)} \hat{\sigma}_{b}^{(j)}\right\rangle_{\hat{\rho}}(a, b=x, y, z)$, respectively, fully specifying all one- and two-qubit reduced density matrices. Product states are parametrized by the orientations $\left\{\boldsymbol{\lambda}_{i}\right\}=\left\{\boldsymbol{n}^{(i)}\right\}$ of each qubit on the Bloch sphere (where $\boldsymbol{n}^{(i)}$ are unit vectors), so that the effective Hamiltonian $H$ describes classical Heisenberg spins (namely, rotators), coupled via bilinear interactions and immersed in an external field:

$$
H\left(\left\{\boldsymbol{n}^{(i)}\right\}\right)=-\sum_{i=1}^{N} \sum_{a=x, y, z} K_{a}^{(i)} n_{a}^{(i)}-\sum_{i<j} \sum_{a, b} K_{a b}^{(i j)} n_{a}^{(i)} n_{b}^{(j)} .
$$

Heisenberg antiferromagnetic chain.-The first example of entangled states that we study with our approach is the thermal equilibrium state of the $S=1 / 2$ Heisenberg chain $\hat{\mathscr{H}}=J \sum_{i=1}^{N} \hat{\boldsymbol{S}}^{(i)} \cdot \hat{\boldsymbol{S}}^{(i+1)}$, where $\hat{\boldsymbol{S}}^{(i)}$ are $S=1 / 2$ spin operators, $J$ is the exchange energy, and periodic boundary conditions (PBCs) are assumed. Thermal equilibrium states $\hat{\rho}\left(\propto \exp \left[-\hat{\mathscr{H}} / k_{B} T\right]\right)$ give $\left\langle\hat{\sigma}_{a}^{(i)}\right\rangle_{\hat{\rho}}=0$ and $\left\langle\hat{\sigma}_{a}^{(i)} \hat{\sigma}_{b}^{(j)}\right\rangle_{\hat{\rho}}=$ $\delta_{a b} C(|i-j|)$, due to rotational invariance of the spin-spin 
couplings and translational invariance. These elementary symmetries of the quantum data are directly inherited by the classical Hamiltonian defining separable states aimed at reproducing them. The Hamiltonian takes the form of a classical long-range Heisenberg model $H\left(\left\{\boldsymbol{n}^{(i)}\right\}\right)=$ $-\sum_{i<j} K_{|i-j|} \boldsymbol{n}^{(i)} \cdot \boldsymbol{n}^{(j)}$ with $K_{r}=K_{N-r}$. The most effective existing multipartite entanglement criterion for these quantum data is based on the collective spin, namely, $\left\langle\hat{\boldsymbol{J}}^{2}\right\rangle=$ $\sum_{i j}\left\langle\hat{\boldsymbol{S}}^{(i)} \cdot \hat{\boldsymbol{S}}^{(j)}\right\rangle<N / 2$ [26,27], which is verified for $t=T / J \lesssim 1.4$. This criterion is a permutationally invariant EW (PIEW), treating correlations at all distances on the same footing, and it cannot be optimal at sufficiently high temperatures, namely, when the correlation length $\xi$ becomes of the order of a few lattice spacings.

As a first validation of our approach, we search for the optimal EW based on two-body correlations $\left\langle\hat{\sigma}_{a}^{(i)} \hat{\sigma}_{a}^{(j)}\right\rangle$ by using as input quantum data the correlations [obtained via quantum Monte Carlo (QMC) calculation [17] ] at $t=1$ for $N=64$ spins, at which $\xi=0.72$. Because of their finite range, we used correlations only up to a distance $r_{\max }=10$.
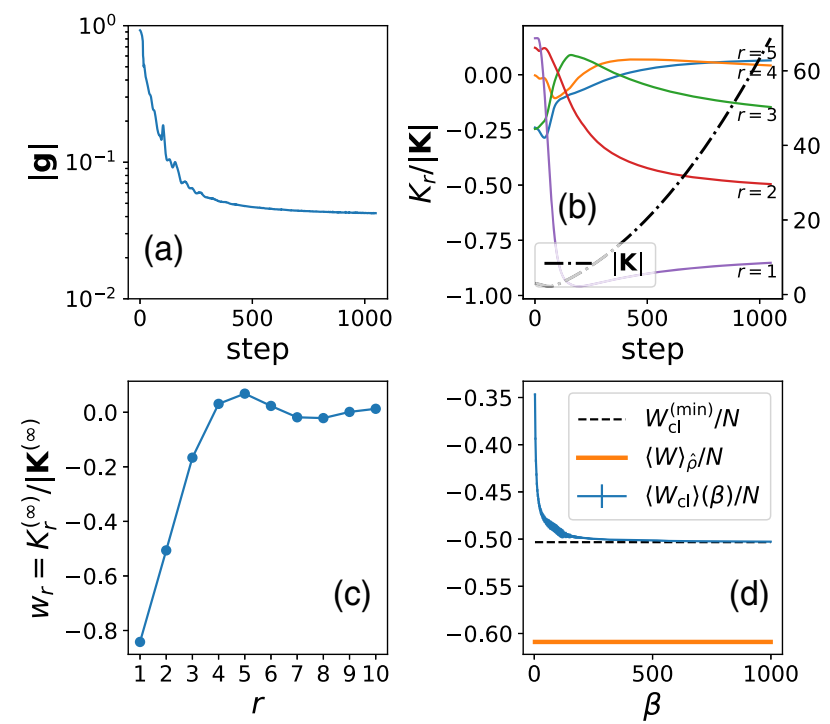

FIG. 2. Data-driven entanglement witness for the Heisenberg chain at $T / J=1$. (a) Distance between the quantum data (all spin-spin correlators) and the optimized separable state ( $g$, gradient of the cost function), as a function of optimization steps in a Nesterov accelerated gradient descent $(\epsilon=0.01)$. Each step contains $10^{5}-10^{7}$ Monte Carlo steps to achieve a relative precision of $10 \%$ on the modulus of the gradient [17]. (b) Normalized coupling constants $K_{r}$ in the classical Hamiltonian defining the separable state (solid lines, left axis) and overall amplitude $|\boldsymbol{K}|$ (dashed-dotted line, right axis). (c) Normalized couplings $K_{r}$ at the end of the algorithm. (d) The separable bound can be obtained via simulated annealing [30] by calculating $\left\langle\mathcal{W}_{\mathrm{cl}}\right\rangle(\beta)$ against $\exp \left[-\beta \mathcal{W}_{\mathrm{cl}}\right]$, ramping $\beta$ from 0 to 1000 . The minimum $\mathcal{W}_{\mathrm{cl}}^{(\min )}$ is actually the lowest value recorded for $\mathcal{W}_{\mathrm{cl}}$ throughout the ramp.
Figure 2 illustrates the results of our approach. The saturation to a finite value of the distance between the quantum data and those of the optimized separable state [measured by the norm of the vector $\boldsymbol{g}$; see Fig. 2(a)] and the divergence of the couplings $K_{r}$ [Fig. 2(b)] clearly indicate the success of entanglement witnessing. The optimal EW operator can be reconstructed, in principle, from the asymptotic value of the gradient vector $\boldsymbol{g}^{(\infty)}$ as $\hat{\mathcal{W}}=-\sum_{i=1}^{N} \sum_{a \in\{x, y, z\}} \sum_{r=1}^{r_{\max }} w_{r} \hat{\sigma}_{a}^{(i)} \hat{\sigma}_{a}^{(i+r)}$ with $w_{r}=$ $-g_{r}^{(\infty)} /\left|g^{(\infty)}\right|$. In practice, we found a more strongly violated EW inequality using the asymptotic couplings of the effective Hamiltonian, namely, $w_{r}=K_{r}^{(\infty)} /\left|\boldsymbol{K}^{(\infty)}\right|-$ which display a clear spatial structure, shown in Fig. 2(c) (see [17] for the numerical values). The final step of the approach consists in determining the separable bound $B_{\text {sep }}=\min _{\hat{\rho}_{p}} \operatorname{Tr}\left(\hat{\rho}_{p} \hat{\mathcal{W}}\right)$. The latter can be obtained as the solution of a set of algebraic equations [28,29]; here, we rather obtain it by finding the ground-state energy of the classical Hamiltonian $\mathcal{W}_{\mathrm{cl}}=-\sum_{i=1}^{N} \sum_{r=1}^{r_{\max }} w_{r} \boldsymbol{n}^{(i)} \cdot \boldsymbol{n}^{(i+r)}$ via temperature annealing [30] [Fig. 2(d)]. We observe that $B_{\text {sep }} / N=-0.5032$, while the quantum data reach $\langle\hat{\mathcal{W}}\rangle_{\hat{\rho}} / N=-0.6089$. In contrast, the best PIEW—properly normalized [17] - is violated by an amount of 0.04552 . This result is not incremental, because the EW inequality we find is optimal among all those containing two-body correlators. Interestingly, for temperatures $t \gtrsim 1.4$ (at which the PIEW ceases to work), we found it numerically impossible to prove that $\hat{\rho}(T)$ is entangled solely based on two-point correlators. This, in turn, shows that the maximal set of thermal states whose entanglement can be witnessed using two-point correlators is essentially captured by the PIEW. This will not be the case in our next example, in which our approach significantly extends the range of witnessed entangled thermal states.

Quantum Ising chain.-Our final example is the quantum Ising model with Hamiltonian $\hat{\mathscr{H}}=-J \sum_{i=1}^{N}\left(\hat{S}_{z}^{(i)} \hat{S}_{z}^{(i+1)}+\right.$ $\left.g \hat{S}_{x}^{(i)}\right)$, where $J$ is the interaction strength and $J g$ the transverse field. In the ground state, the system displays a quantum critical point (QCP) at $g=g_{c}=1 / 2$ between a ferromagnetic phase $\left(g<g_{c}\right)$ and a paramagnetic phase $\left(g>g_{c}\right)$ [31]. At finite temperatures around the QCP, the system is known to exhibit robust entanglement [32-34]. Given the symmetries of the correlation functions $\left(\left\langle\hat{\sigma}_{a}^{(i)}\right\rangle_{\hat{\rho}}=0\right.$ for $\left.a=y, z ;\left\langle\hat{\sigma}_{a}^{(i)} \hat{\sigma}_{b}^{(j)}\right\rangle_{\hat{\rho}} \sim \delta_{a b}\right)$, the classical Hamiltonian tailored to reproduce them is of the form $H\left(\left\{\boldsymbol{n}^{(i)}\right\}\right)=-K_{x} \sum_{i=1}^{N} n_{x}^{(i)}-\sum_{a=x, y, z} \sum_{i<j} K_{a}^{|i-j|} n_{a}^{(i)} n_{a}^{(j)}$. As input quantum data, we consider the correlation functions of a chain of $N=64$ spins with PBCs at a temperature $t=T / J=0.28$ for $g=0.5$-obtained as well via QMC. Given the finite correlation length, we used correlators only up to a distance $r_{\max }=20$. Following the same procedure as the one described for the Heisenberg 


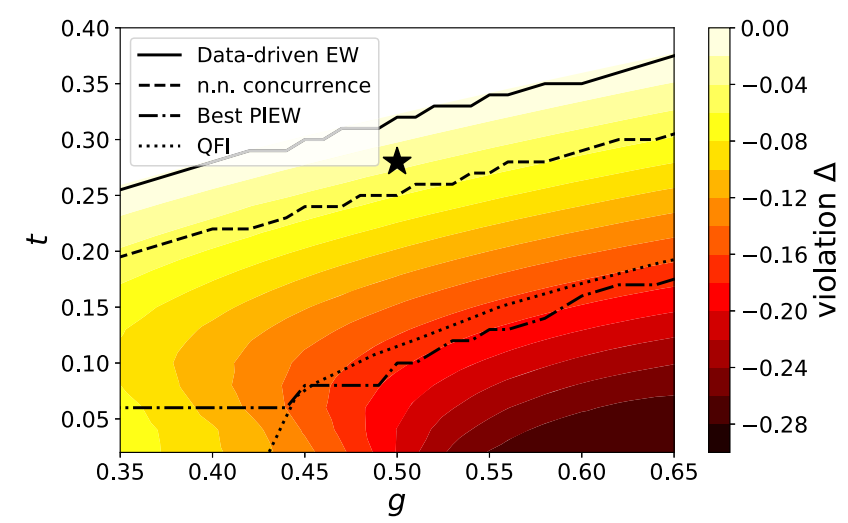

FIG. 3. Data-driven EW for the quantum Ising chain. Phase diagram around the QCP. The star corresponds to $t=0.28$ and $g=0.5$, at which the quantum data used as input were calculated. The color represents the violation $\Delta=\left(\langle\hat{\mathcal{W}}\rangle_{\hat{\rho}}-B_{\text {sep }}\right) / N$ of our data-driven EW. The various curves correspond to the temperature below which different entanglement criteria are satisfied [nearest-neighbor concurrence [35], best PIEW [12], and quantum Fisher information (QFI) of $\left.\hat{J}_{z}[32]\right]$.

chain, we find an optimal EW operator which is spatially structured, of the form $\hat{\mathcal{W}}=-w_{x} \sum_{i=1}^{N} \hat{\sigma}_{x}^{(i)}-$ $\sum_{a=x, y, z} \sum_{i<j} w_{a}^{(|i-j|)} \hat{\sigma}_{a}^{(i)} \hat{\sigma}_{a}^{(j)}$ (coefficients and separable bound in the Supplemental Material [17]). In Fig. 3, we show that this new EW criterion, optimal for the thermal state at $t=0.28$ and $g=0.5$, allows one to prove entanglement for a larger set of thermal states than all the existing criteria in the literature (namely, the nearestneighbor concurrence [35], the PIEW [12], and the quantum Fisher information [32]—see [17] for further details).

Conclusions.-We introduced a data-driven method to probe multipartite entanglement in many-body systems. This method relies on mapping separable states onto Boltzmann distributions for a classical field theory on a lattice. The classical degrees of freedom of this field theory are dictated by the considered partitioning of the system. The structure of the corresponding classical Hamiltonian is dictated by the quantum data at hand, and its parameters are optimized in order to fit at best the quantum data. This method allows one to exhaustively test the entanglement witnessing capability of a set of quantum data in a time scaling polynomially with the number of parties in the partition (if the size of quantum data is also polynomial); this is guaranteed whenever the classical field theory is not a model of a glass (namely, when it does not feature disorder and frustration). This opens the way to the systematic certification of entanglement in intermediatescale quantum devices.

We are very grateful to Antonio Acín for insightful discussions. I. F. acknowledges support from the Government of Spain (FIS2020-TRANQI and Severo
Ochoa CEX2019-000910-S), Fundació Cellex and Fundació Mir-Puig through an ICFO-MPQ postdoctoral fellowship, Generalitat de Catalunya (CERCA, AGAUR SGR 1381, and QuantumCAT), and MINECO-EU QuantERA MAQS [funded by The State Research Agency (AEI) PCI2019-111828-2/10.13039/ 501100011033]. T. R. acknowledges support from ANR (EELS project) and QuantERA (MAQS project). Numerical computations have been performed at the Pôle Scientifique de Modélisation Numérique (PSMN).

*irenee.frerot@gmail.com tommaso.roscilde@ens-lyon.fr

[1] I. M. Georgescu, S. Ashhab, and F. Nori, Quantum simulation, Rev. Mod. Phys. 86, 153 (2014).

[2] J. Preskill, Quantum computing and the entanglement frontier, arXiv:1203.5813.

[3] F. Fröwis, P. Sekatski, W. Dür, N. Gisin, and N. Sangouard, Macroscopic quantum states: Measures, fragility, and implementations, Rev. Mod. Phys. 90, 025004 (2018).

[4] M. Paris and J. Rehacek, Quantum State Estimation, Lecture Notes in Physics (Springer, Berlin, 2004).

[5] A. C. Doherty, P. A. Parrilo, and F. M. Spedalieri, Detecting multipartite entanglement, Phys. Rev. A 71, 032333 (2005).

[6] O. Gühne, M. Reimpell, and R. F. Werner, Lower bounds on entanglement measures from incomplete information, Phys. Rev. A 77, 052317 (2008).

[7] M. Navascues, F. Baccari, and A. Acín, Entanglement marginal problems, arXiv:2006.09064.

[8] R. F. Werner, Quantum states with einstein-podolsky-rosen correlations admitting a hidden-variable model, Phys. Rev. A 40, 4277 (1989).

[9] O. Gühne and G. Tóth, Entanglement detection, Phys. Rep. 474, 1 (2009).

[10] I. Frérot and T. Roscilde, Detecting Many-Body Bell NonLocality by Solving Ising Models, Phys. Rev. Lett. 126, 140504 (2021).

[11] Otherwise, one can always reproduce the correlations among these observables using separable states by choosing the local states $\left|\psi_{i}\right\rangle$ to be joint eigenstates of the observables and reproducing with $p$ the classical correlations among the observables.

[12] G. Tóth, C. Knapp, O. Gühne, and H. J. Briegel, Spin squeezing and entanglement, Phys. Rev. A 79, 042334 (2009).

[13] G. Vitagliano, P. Hyllus, I. N. L. Egusquiza, and G. Tóth, Spin Squeezing Inequalities for Arbitrary Spin, Phys. Rev. Lett. 107, 240502 (2011).

[14] $d_{i}$ complex amplitudes, up to normalization of the state and an arbitrary global phase.

[15] H. C. Nguyen, R. Zecchina, and J. Berg, Inverse statistical problems: from the inverse Ising problem to data science, Adv. Phys. 66, 197 (2017).

[16] E. T. Jaynes, Information theory and statistical mechanics, Phys. Rev. 106, 620 (1957).

[17] See Supplemental Material at http://link.aps.org/ supplemental/10.1103/PhysRevLett.127.040401 for a discussion of (i) further details of the optimization algorithm 
and its relationship with existing complexity results; (ii) the quantum data from quantum Monte Carlo calculations; and (iii) the existing entanglement witnesses for the quantum Ising chain, which includes Refs. [18-25].

[18] S. Boyd and L. Vandenberghe, Convex Optimization (Cambridge University Press, Cambridge, England, 2004).

[19] L. Gurvits, Classical deterministic complexity of Edmonds' problem and quantum entanglement, in Proceedings of the Thirty-Fifth Annual ACM Symposium on Theory of Computing, STOC '03, 1019 (Association for Computing Machinery, New York, 2003), https://doi.org/10.1145/ 780542.780545 .

[20] F. Barahona, On the computational complexity of ising spin glass models, J. Phys. A 15, 3241 (1982).

[21] M. Creutz, Overrelaxation and Monte Carlo simulation, Phys. Rev. D 36, 515 (1987).

[22] O. F. Syljuåsen and A. W. Sandvik, Quantum Monte Carlo with directed loops, Phys. Rev. E 66, 046701 (2002).

[23] M. Boll, T. A. Hilker, G. Salomon, A. Omran, J. Nespolo, L. Pollet, I. Bloch, and C. Gross, Spin- and density-resolved microscopy of antiferromagnetic correlations in fermihubbard chains, Science 353, 1257 (2016).

[24] A. Browaeys and T. Lahaye, Many-body physics with individually controlled rydberg atoms, Nat. Phys. 16, 132 (2020).

[25] L. Pezzè and A. Smerzi, Quantum theory of phase estimation, in Proceedings of the International School of Physics
"Enrico Fermi", edited by G. Tino and M. Kasevich (IOS691 Press, Amsterdam, 2014), p. 691.

[26] G. Tóth, Entanglement detection in optical lattices of bosonic atoms with collective measurements, Phys. Rev. A 69, 052327 (2004).

[27] M. Wieśniak, V. Vedral, and Č Brukner, Magnetic susceptibility as a macroscopic entanglement witness, New J. Phys. 7, 258 (2005).

[28] J. Sperling and W. Vogel, Multipartite Entanglement Witnesses, Phys. Rev. Lett. 111, 110503 (2013).

[29] S. Gerke, W. Vogel, and J. Sperling, Numerical Construction of Multipartite Entanglement Witnesses, Phys. Rev. X 8, 031047 (2018).

[30] S. Kirkpatrick, C. D. Gelatt, and M. P. Vecchi, Optimization by simulated annealing, Science 220, 671 (1983).

[31] S. Sachdev, Quantum Phase Transitions (Cambridge University Press, Cambridge, England, 2011).

[32] P. Hauke, M. Heyl, L. Tagliacozzo, and P. Zoller, Measuring multipartite entanglement through dynamic susceptibilities, Nat. Phys. 12, 778 (2016).

[33] I. Frérot and T. Roscilde, Quantum Critical Metrology, Phys. Rev. Lett. 121, 020402 (2018).

[34] I. Frérot and T. Roscilde, Reconstructing the quantum critical fan of strongly correlated systems via quantum correlations, Nat. Commun. 10, 577 (2019).

[35] W. K. Wootters, Entanglement of Formation of an Arbitrary State of Two Qubits, Phys. Rev. Lett. 80, 2245 (1998). 